University of Nebraska - Lincoln

DigitalCommons@University of Nebraska - Lincoln

$1-2004$

\title{
The hemolymph $\mathrm{JH}$ titer exhibits a large-amplitude, morph- dependent, diurnal cycle in the wing-polymorphic cricket, Gryllus firmus
}

Zhangwu Zhao

University of Nebraska - Lincoln

Anthony J. Zera

University of Nebraska - Lincoln, azera1@unl.edu

Follow this and additional works at: https://digitalcommons.unl.edu/bioscizera

Part of the Microbiology Commons

Zhao, Zhangwu and Zera, Anthony J., "The hemolymph JH titer exhibits a large-amplitude, morphdependent, diurnal cycle in the wing-polymorphic cricket, Gryllus firmus" (2004). Anthony Zera Publications. 31.

https://digitalcommons.unl.edu/bioscizera/31

This Article is brought to you for free and open access by the Papers in the Biological Sciences at DigitalCommons@University of Nebraska - Lincoln. It has been accepted for inclusion in Anthony Zera Publications by an authorized administrator of DigitalCommons@University of Nebraska - Lincoln. 
Published in Journal of Insect Physiology 50:1 (January 2004), pp. 93-102; doi 10.1016/j.jinsphys.2003.10.003

Copyright ( 2003 Elsevier Ltd. Used by permission. http://www.sciencedirect.com/science/journal/00221910

Submitted June 19, 2003; revised October 10, 2003; accepted October 13, 2003; published online December 2, 2003.

\title{
The hemolymph JH titer exhibits a large-amplitude, morph-dependent, diurnal cycle in the wing-polymorphic cricket, Gryllus firmus
}

\author{
Zhangwu Zhao and Anthony J. Zera \\ School of Biological Sciences, University of Nebraska, Lincoln, NE 68588-0118, USA \\ Corresponding author-A. J. Zera, tel 402 472-2768, fax 402 472-2083, email azera1@unl.edu
}

\begin{abstract}
The hemolymph juvenile hormone (JH) titer was measured in over 500 flight-capable and flightless, adult female Gryllus firmus at 3-6 h intervals during each of days 2-8 of adulthood. The flight-capable morph exhibited a large-amplitude daily cycle in the hemolymph JH titer, while the flightless morph exhibited a barely perceptible cycle. The JH titer cycle was observed on all days in the flight-capable morph, but the large amplitude cycle (>15-20 fold increase in mean titer; $>100$-fold increase in some individuals), began on day 5. For both the large and small amplitude cycles, the JH titer peaked near the end of the photophase-beginning of the scotophase. The hemolymph ecdysteroid titer did not exhibit a corresponding large amplitude daily cycle, although a low amplitude cycle (1-3-fold change) was seen in both morphs. The large magnitude rise in the JH titer in the flight-capable morph during the photophase was not due to decreased hemolymph volume or JH degradation. Daily cycles in the JH titer may be common, but may have gone unnoticed in other insect species due to restricted temporal sampling. Failure to identify these cycles can result in substantial errors in inferring biological roles for JH. Because JH regulates flight behaviors, morph-specific daily cycles in the JH titer may be especially common in dispersal-polymorphic insects, in which flight is restricted to one morph during a limited period of the day or night. However, because JH regulates numerous biological traits, analogous cycles may be common in insects exhibiting other types of complex (e.g. caste or phase) polymorphism, in which morphs differ in a biological characteristic that is restricted to a specific period of the photophase or scotophase.
\end{abstract}

Keywords: juvenile hormone, JH, JH titer,dispersal, circadian rhythm, wing polymorphism

\section{Introduction}

Wing polymorphism occurs widely in the Insecta where it plays a central role in the life cycle of many species (Dingle, 1996, Zera and Denno, 1997, and Zera and Harshman, 2001). The polymorphism consists of one or more flightless morphs with underdeveloped wings and flight muscles or underdeveloped flight muscles alone, and a flight-capable morph with fully developed wings and flight muscles (Zera et al., 1997). Importantly, flight capability trades off (is negatively associated) with ovarian growth: flightless adults begin egg development earlier and typically produce considerably more eggs than their flight-capable counterpart during early adult- hood (Dingle, 1996, Zera and Denno, 1997, and Zera and Harshman, 2001). Wing polymorphism has been used extensively by evolutionary biologists as a model to study the evolution of dispersal, development, and life histories and by insect physiologists to study the endocrine regulation of alternate developmental pathways within species (Hardie and Lees, 1985, Nijhout, 1994, Dingle, 1996, Zera and Denno, 1997, and Zera and Harshman, 2001).

Several decades ago, Wigglesworth (1961) and Southwood (1961) proposed that the development of alternate morphs is regulated by differences in the $\mathrm{JH}$ titer during the juvenile stage, with an elevated $\mathrm{JH}$ titer specifying the flightless, short-winged or wingless morphs. 
Since JH positively affects many aspects of reproduction (Nijhout, 1994 and Wyatt and Davey, 1996), an elevated $\mathrm{JH}$ titer in flightless adults would also account for their earlier and greater egg production (Zera and Denno, 1997 and Zera and Harshman, 2001). This hypothesis has been widely accepted and presented as the standard model in general discussions of the endocrine regulation and evolution of wing polymorphism (e.g. Gould, 1977, Matsuda, 1987, Nijhout, 1994, and Nijhout, 1999). However, most of the data supporting the aforementioned hypothesis are indirect, having been obtained from hormone manipulation studies. Few studies have directly compared hemolymph $\mathrm{JH}$ titers between flight-capable and flightless morphs during either juvenile or adult stages (Zera et al., 1989, Zera and Denno, 1997, and Zera and Cisper, 2001).

In a recent study, Zera and Cisper, (2001) reported that differences in the hemolymph $\mathrm{JH}$ titer between adult morphs were more complex than proposed by the classic JH-wing-morph hypothesis described above. Specifically, they found that the JH titer was temporally constant in the SW (short-winged, flightless) morph, but rose 10-fold in the flight-capable LW(f) morph (longwinged with functional flight muscles) between the early and late portions of the photophase. This dramatic temporal variation resulted in the $\mathrm{JH}$ titer being higher in the SW vs. $L W(f)$ morph during the early photophase, but lower in the SW vs. the $L W(f)$ morph during the late photophase. Thus, no morph has a consistently higher $\mathrm{JH}$ titer than the other morph during all times of the day. These results have important implications for models of the endocrine regulation of wing polymorphism, as well as many general issues in $\mathrm{JH}$ endocrinology (Zera and Cisper, 2001 and Zera and Harshman, 2001; see Discussion).

The previous study of Zera and Cisper (2001) mainly focused on JH titer differences between replicate LW(f) and SW-selected lines of G. firmus and only measured $\mathrm{JH}$ titers at two points in the photophase on two, nonconsecutive days of adulthood. Thus, numerous questions concerning morph-dependent diurnal hormone titer changes were unanswered. Is $\mathrm{JH}$ the only hormone whose titer changes diurnally in the LW(f) but not in the $\mathrm{SW}$ morph? What is the duration of the elevated JH titer in the the LW(f) morph? A few hours? Most of the scotophase? Does the JH titer cycle on a daily basis in the LW(f) morph and on which day does the cycle begin? What are the proximate mechanisms that regulate the morph-dependent differences in the JH titer? The goals of the present study were to obtain a more detailed and expanded picture of daily $\mathrm{JH}$ titer changes in flight-capable [LW(f)] and flightless (SW) females by measuring the JH titer at 3-6 h intervals on each of 7 consecutive days during the first week of adulthood. We also measured the ecdysteroid titer during these periods to de- termine if morph-dependent changes also occurred in the titers of other hormones. Finally, we measured several aspects of $\mathrm{JH}$ degradation and hemolymph volume during early and late portions of the photophase to determine if the large-magnitude, morph-dependent rise in the JH titer was due to morph-dependent changes in either of these factors.

\section{Materials and methods}

\subsection{Chemicals}

All chemicals and solvents used in the present study were at least reagent or HPLC grade and were purchased from Sigma Chemical Company, Mallinckrodt, or Fisher Scientific. Racemic unlabeled JH III and silica gel thin-layer chromatographic plates were purchased from Sigma Chemical Co. $\left[{ }^{3} \mathrm{H}\right]$-juvenile hormone III (19.5 Ci/mmol), $\left[{ }^{3} \mathrm{H}\right]$-ecdysone $(85 \mathrm{Ci} / \mathrm{mmol})$, and $\left[{ }^{14} \mathrm{C}\right]-$ inulin $(14.3 \mathrm{mCi} / \mathrm{mmol})$ were purchased from New England Nuclear.

\subsection{Insects, morph designations and rearing conditions}

Gryllus firmus, the sand cricket, occurs in the southeastern United States as a long-winged (LW) morph, some of which are capable of flight, or as a short-winged (SW) form that is obligatorily flightless (Veazy et al., 1976). Except for a few rare cases, all SW females have white, non-functional flight muscles which never fully develop. All LW females initially have large, pink flight muscles at the adult molt and are denoted as LW(f). After about 5-6 days of adulthood, some LW(f) individuals begin to histolyze their flight muscles thus becoming flightless (denoted as LW(h); see Zera et al. [1997]). The G. firmus used in the present study were derived from a pair of LW- and SW-selected lines (Block-2; see Zera and Cisper [2001]) that were derived from a colony founded from 30 gravid females collected in Gainesville, Florida during the summer of 1995.

Crickets were reared under a 16:8 L:D cycle at $28^{\circ} \mathrm{C}$ and were fed the standard diet described in Zera and Larsen (2001). Other details of rearing can be found in Zera and Cisper (2001). Crickets used for experiments were checked for molting at 24-h intervals and equal numbers of newly molted males and females were housed together at a density of six per 1-gallon box or 12 per 3-gallon box without oviposition material. Oviposition material was not provided so that total mass of eggs produced by individual females could be measured by weighing the ovaries. After about day 5 , the ovaries are large in SW females (see Results). JH titers were measured on days 7 and 8 on a separate group of crickets which had been raised as described above, but which had access to oviposition substrate (moist cot- 
ton) on all days. This was done to determine if forced retention of eggs had any effect on the JH titer after day 6.

\subsection{Hemolymph collection and hormone extraction}

A minimum of 450-550 LW(f) and SW G. firmus were assayed for hemolymph $\mathrm{JH}$ and ecdysteroid titers, ovarian mass, and flight muscle phenotype at $6 \mathrm{~h}$ intervals on days 2-8 of adulthood (3-h intervals on day 5). Because of their low frequency during early adulthood, females with histolyzed flight muscles [LW(h)] were not studied. Ten to $20 \mu \mathrm{l}$ hemolymph from an individual female G. firmus were collected in micropipettes from cuts in the cerci and legs. Hemolymph was blown into a test tube containing $300 \mu 190 \%$ methanol in water. Samples were sonicated for 3-5 s and JH was extracted twice with $600 \mu \mathrm{l}$ hexane. Hexane extracts were stored in glass vials at $-80{ }^{\circ} \mathrm{C}$ until radioimmunassay (RIA). Methanol extracts were also saved under the same conditions for subsequent ecdysteroid-RIA. Bled females were stored at $-20^{\circ} \mathrm{C}$ until dissection when reproductive characteristics and flight muscle phenotype were determined. Background experiments showed that these features did not change during the time the crickets were frozen.

\subsection{Juvenile hormone and ecdysteroid RIAs}

The juvenile hormone titer was measured in triplicate, on an aliquot of a hexane extract of hemolymph from an individual adult female G. firmus. We used an RIA employing a polyclonal antiserum that is specific for the naturally occurring (10R) enantiomer of juvenile hormone III (Hunnicutt et al., 1989 and Huang et al., 1994). This antiserum was kindly provided by D.W. Borst (Illinois State University). JH III has been identified as the only juvenile hormone in G. firmus (Yadlowski, 1994) and other Gryllus species (Loher et al., 1983 and Zera et al., 1989). Assay conditions and validation of the assay are described in Cisper et al. (2000). Most importantly, Cisper et al. (2000) demonstrated that this JH-RIA is specific for JH-III in crude hemolymph extracts: when a hexane extract of $G$. firmus hemolymph was fractionated via HPLC, all immunreactivity co-eluted with a JH-III standard. Furthermore, JH titer estimates were not significantly affected by lipid in the hemolymph, thus allowing the RIA to be performed on unpurified hexane extracts of hemolymph. This RIA also has been used to reliably estimate the JH titer in unpurified hexane extracts of hemolymph of several other insect species (e.g. Huang et al., 1994 and Trumbo et al., 1995). The ecdysteroid RIA was performed as described previously on a small aliquot of the methanol extract (three replicates) of hemolymph from an individual G. firmus (Zera and
Bottsford, 2001), using the polyclonal ecdysteroid antiserum from the "A" series of Horn et al. (1976). This antiserum was produced in the laboratory of W. E. Bollenbacher (University of North Carolina, Chapel Hill), and was kindly distributed by E. S. Chang (Bodega Marine Laboratory, Bodega Bay, California). Details of the statistical procedures used to estimate $\mathrm{JH}$ and ecdysteroid titers (non-linear regression) can be found in Cisper et al. (2000) and Zera and Bottsford (2001).

\subsection{Hemolymph volume, hemolymph JHE activity, and whole-cricket JH degradation}

Hemolymph volume was determined by the standard $\left[{ }^{14} \mathrm{C}\right]$-inulin dilution method, as applied to Gryllus (Zera and Holtmeier, 1992). Briefly, $\left[{ }^{14} \mathrm{C}\right]$-inulin in saline was injected into the hemocoel of a cricket, and allowed to equilibrate within the organism for 10-20 min. A small hemolymph sample was taken and DPM determined. Hemolymph volume was estimated from the degree of dilution of injected inulin. Activity of the two main JHdegrading enzymes, juvenile hormone esterase (JHE) and juvenile hormone epoxide hydrolase (JHEH) were quantified using standard radiochemical assays as described in Zera and Huang (1999). JHE is the sole JH-degrading enzyme in the hemolymph, while both JHE and JHEH are found in many non-hemolymph tissues. We measured hemolymph JHE activity, and whole-cricket JHE and JHEH activities.

\subsection{Ovarian masses and flight muscle phenotype}

Both ovaries were dissected and their wet masses recorded for all individuals whose $\mathrm{JH}$ or ecdysteroid titers were measured. Flight muscle phenotype (white, reduced; non-functional vs. pink, large, functional) was recorded for all G. firmus.

\section{Results}

\subsection{JH titer}

The mean JH titer exhibited a dramatic morph-dependent daily cycle when sampled at 3-6-h intervals. The titer rose 15-20-fold late in the photophase and dropped back down to near baseline levels by the early scotophase in the LW(f) morph, while the JH titer in the SW morph exhibited a barely perceptible daily fluctuation (Figure 1). In the LW(f) morph, the cycle was first evident on day 2 and was observed on every day thereafter. The amplitude of the titer cycle was low on days 2-4 (1-3-fold change over baseline), but became much larger on days 5-7 (15-20-fold change). The large-amplitude peak occurred at $1-4 \mathrm{~h}$ prior to lights off, the last points in the photophase that were sampled. Results described 


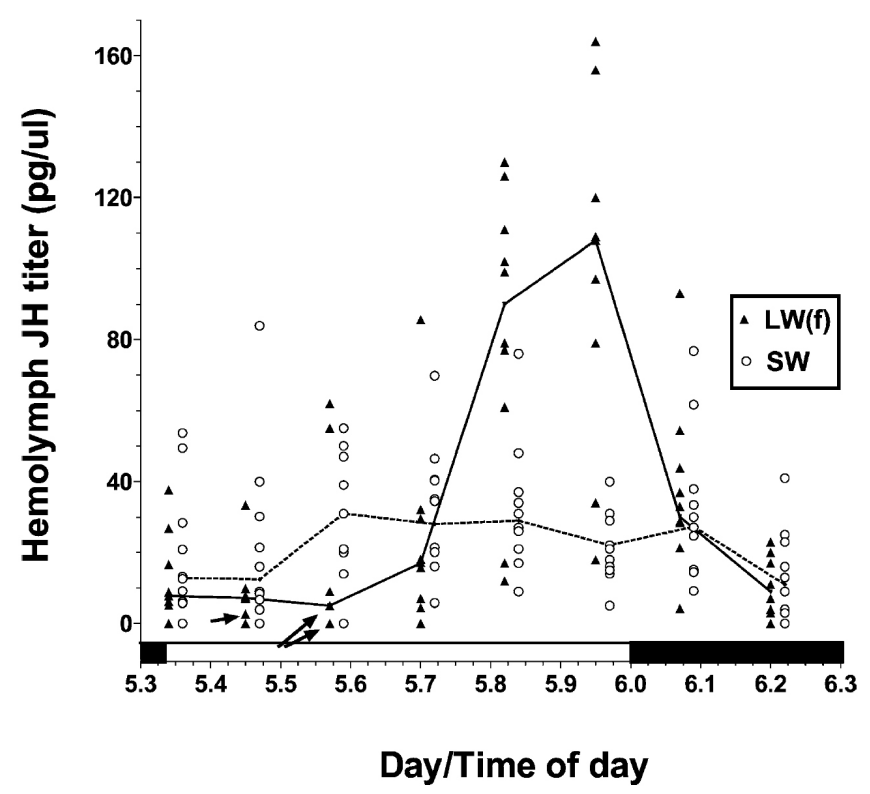

Figure 1. Mean \pm SEM hemolymph juvenile hormone titer $(\mathrm{JH})$ in adult female wing morphs of Gryllus firmus as a function of adult age and time of day. Crickets were reared under a 16L:8D photoperiod, with the duration of the photophase and scotophase denoted by open and blackened bars, respectively, on the $\mathrm{x}$-axis. Numbers on $\mathrm{X}$-axis refer to day since molt to adulthood (=day 0$)$ and proportion of the day that had passed (starting at lights off). Titers were measured every $6 \mathrm{~h}$ (every $3 \mathrm{~h}$ on day 5), beginning at $2 \mathrm{~h}$ after lights off. LW(f), flight capable morph, with long wings and fully-developed flight muscles; SW, flightless morph, with short-wings and underdeveloped, non-functional flight muscles. Females were not provided with oviposition material. Means were based on titer determinations of 8-10 individuals, except for the following days/times-of-day where sample sizes were $4-5: 2.08,3.08,3.83$, and 7.58 . JH titers in individual females on day 5 are presented in Figure 3.

above were for females not provided with oviposition material. The JH titer daily cycle was virtually identical for 7-8-day-old LW(f) adult females that were provided with oviposition material (Figure 2A).

The JH titer exhibited a much more moderate cycle in SW non-ovipositing females: the amplitude was considerably smaller (less than 3-fold change), and the cycle started a few days later (day 4) than in LW(f) females (day 2; Figure 1). In 7-8-day-old SW females provided with oviposition material, even the modest increase in the JH titer during the latter part of the photophase ( Figure 1) was not seen ( Figure 2A). Mean JH titer ( Figure 1) was moderately but consistently higher (15 of 18 cases; $\chi^{2}$ test: $P<0.05$ ) in SW vs. LW(f) females from day 2 until the beginning of the large-amplitude increase in the JH titer in LW(f) females near the end of the photophase on day 5 .

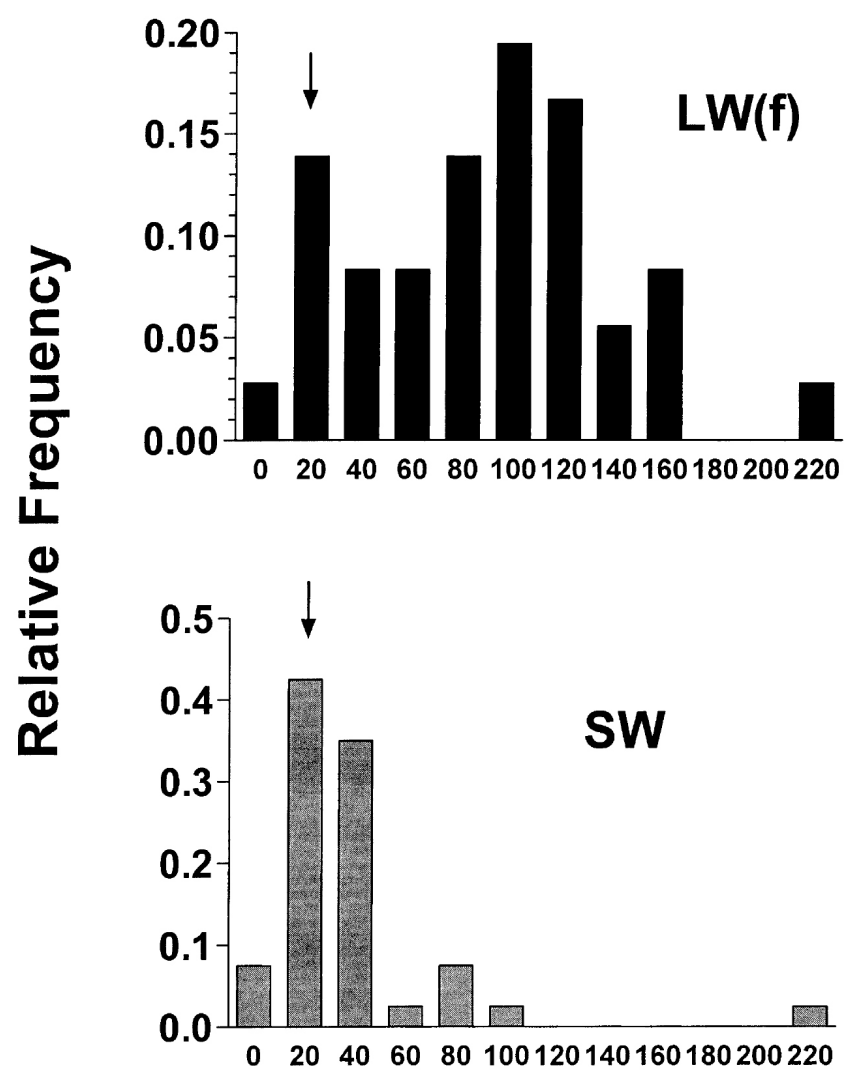

\section{JHtiter (pg/ul)}

Figure 2. Hemolymph $\mathrm{JH}$ titer (A), hemolymph ecdysteroid titer (B), and ovarian mass (C) measured on days 7 and 8 of adulthood in LW(f) and SW females provided with oviposition material. Symbols and axes are the same as in Figure 1. Note the virtually identical morph-dependent JH titer cycle in LW(f) females as is seen in Figure 1 for $\operatorname{LW}(\mathrm{f})$ females that were not allowed to oviposit.

Figure 3 illustrates the $\mathrm{JH}$ titer in individual $\mathrm{LW}(\mathrm{f})$ and SW females measured at 3-h intervals throughout a $24 \mathrm{~h}$ cycle on days 5-6 of adulthood. At the JH titer peak, individual hemolymph titers were more than 80-fold higher (160-120 pg/ $\mu \mathrm{l}$; 1-4 h prior to lights-off) than those at baseline levels $(<2 \mathrm{pg} / \mu \mathrm{l} ; 6 \mathrm{~h}$ after lights on). Most of the rise and fall in the JH titer in the LW(f) morph occurred very rapidly, within a 3 -h period in each case (Figure 3). The JH titer was elevated in this morph between 3-7 h of the 24-h cycle. Distributions of the JH titer in day 5-7 LW(f) and SW females during the last $4 \mathrm{~h}$ of the photophase, when the JH titer was substantially elevated in the LW(f) morph, are presented in Figure 4. Among-individual variation in the JH titer was 


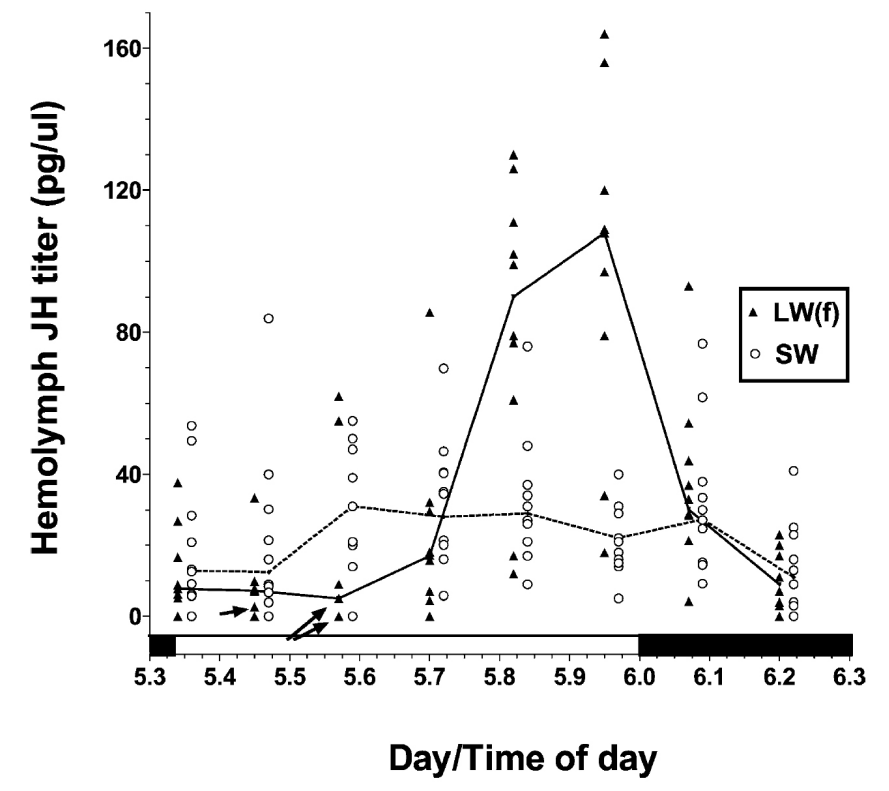

Figure 3. Hemolymph JH titer in individual female G. firmus measured at $3 \mathrm{~h}$ intervals during a $24 \mathrm{~h}$ cycle on days 5-6 of adulthood. Lines connect median values. Morph symbols, and scale on the $\mathrm{x}$-axis are defined in Figure 1. To improve clarity, titer points are offset slightly before and after sampling time. For time 5.58 in the LW(f) morph, the two lowest triangles (denoted by arrows) each represent three nearly-identical values; for time 5.46, the second-lowest triangle for the LW(f) morph represents three nearly-identical values.

much greater for $\mathrm{LW}(\mathrm{f})$ than for SW females. LW(f) females exhibited a bimodal distribution of JH titers (120 and $30 \mathrm{pg} / \mu \mathrm{l}$ ) and the lower mode was similar to the mode in the unimodal distribution of the JH titer in SW females (Figure 4).

\subsection{Ecdysteroid titer}

In the flight-capable LW(f) and flightless SW morphs, the average ecdysteroid titer varied much less during a 24-h cycle (about 2-3-fold) than did the JH titer (1520-fold) [compare Figure 5 and Figure 2 (ecdysteroid titer) with Figure 1 and Figure 2 (JH titer). No morphdependent diurnal variation was observed, but the ecdysteroid titer did exhibit a parallel daily cycle in both morphs on days 5-7. On these days, the ecdysteroid titer peak occurred near the end of the photophase-beginning of the scotophase, which roughly coincided with the JH titer peak (compare Figure 5 and Figure 2 with Figure 1 and Figure 2). The mean ecdysteroid titer averaged over days 2-7 of adulthood (data from Figure 5) was approximately $30 \%$ higher in SW $(0.21 \mathrm{ng} / \mu \mathrm{l})$ vs. $\mathrm{LW}(\mathrm{f})$ females $(0.16 \mathrm{ng} / \mu \mathrm{l}$; least-squares means computed from ANOVA). The titer of this hormone was consistently higher in temporal samples of SW vs. LW(f) females during this time (i.e. higher in 21 out of 26 temporal samples; sign test: $\left.\chi^{2}=5.44, P<0.02\right)$.

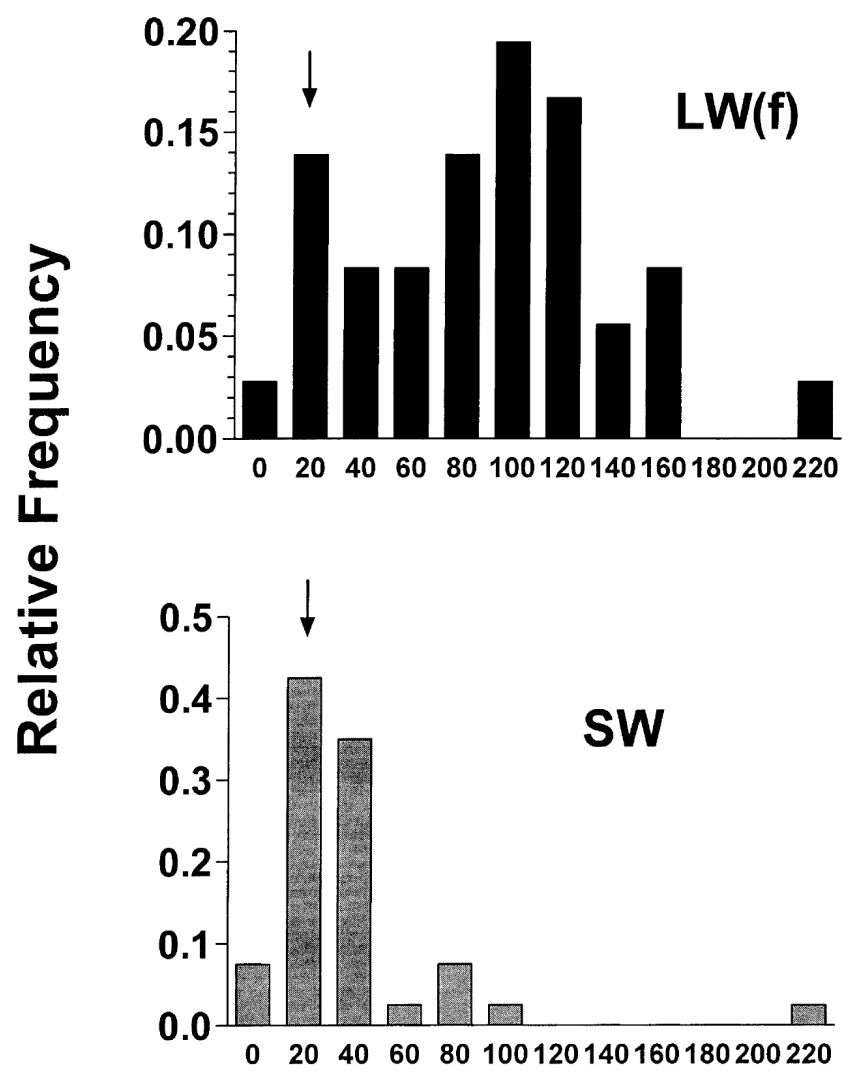

JHtiter (pg/ul)

Figure 4. Distributions of the hemolymph JH titer in individual $\mathrm{LW}(\mathrm{f})$ and SW G. firmus during the period of the photophase when the JH titer was substantially elevated in the LW(f) morph (last $4 \mathrm{~h}$ before lights off on days 5-7). Morph designations are defined in Figure 1. Note the bimodal distribution of titers in LW(f) females, the lower mode of which corresponds with the mode in the unimodal distribution of titers in SW females. Each distribution was based on JH titer determinations in $49 \mathrm{LW}(\mathrm{f})$ and 49 SW females.

\subsection{Ovarian mass}

In the main study (no oviposition material), ovarian mass, scaled to whole-body wet mass, was significantly greater in SW than in LW(f) females on nearly all temporal samples between days 3 and 8 (Figure 5B). Since no oviposition material had been provided to these females, these differences reflect substantially faster ovarian growth in SW vs. LW(f) females. Even when morphs were allowed to oviposit, ovarian mass was still larger in SW in than in LW(f) females ( Figure 2C). No obvious morph-dependent diurnal cycles were observed for ovarian mass.

\subsection{Hemolymph volume}

Hemolymph volume, as a percent of whole-body wet mass, was approximately $25-30 \%$ larger in SW than in 
LW(f) females on day 5 of adulthood (Table 1; ANCOVA of hemolymph volume with whole-body wet mass as the covariate: $P=0.078$ ). There was a highly significant decrease in hemolymph volume between the early (within $1 \mathrm{~h}$ after lights on) and late (4 $\mathrm{h}$ prior to lights off) portions of the $16 \mathrm{~h}$ photophase (ANCOVA: $P=0.014$ ). The decrease occurred to a similar degree in both morphs: morph $\times$ time-of-day interaction for hemolymph volume was non-significant $(P=0.81)$. Similar results were observed on day 7 of adulthood in the $\mathrm{LW}(\mathrm{f})$ and SW lines and on days 5 and 7 in other lines (data not shown).

\subsection{JHE and JHEH activities in hemolymph and whole-crickets}

Whole-cricket JH-epoxide hydrolase activity did not differ significantly between morphs or time-of-day (Table $1)$. Whole-cricket JH esterase activities were about 23-fold higher in SW females compared with LW(f) females both early and late in the photophase, and only increased minimally between these two time points. Hemolymph JH esterase activities were slightly higher in LW(f) than in SW females, and increased only slightly from samples early and late in the photophase.

\subsection{Correlations between JH titer, ecdysteroid titer and ovar- ian mass}

For samples taken in the main study, $\mathrm{JH}$ and ecdysteroid titers were uncorrelated $(r=0.18, P>0.1$, n.s.; $n=$ $420)$. However, the titer of each hormone was weaklymoderately correlated with ovarian mass (JH: $r=0.20$, $P<0.01$; ecdysteroid: $r=0.37, P<0.01$ ). With one exception, correlations did not change significantly when they were measured in either morph alone, or within a specific time-of-day (data not shown). That exception was late in the photophase (1-4 h prior to lights off on days 5-7), when the JH titer peaked in the LW(f) morph. At that time of the photoperiod, the JH titer was uncorrelated with ovarian mass $(r=-0.04, P>0.1$, n.s.; $n=99)$, although ovarian mass and the edysteroid titer still remained moderately correlated $(r=0.39, P<0.01)$.
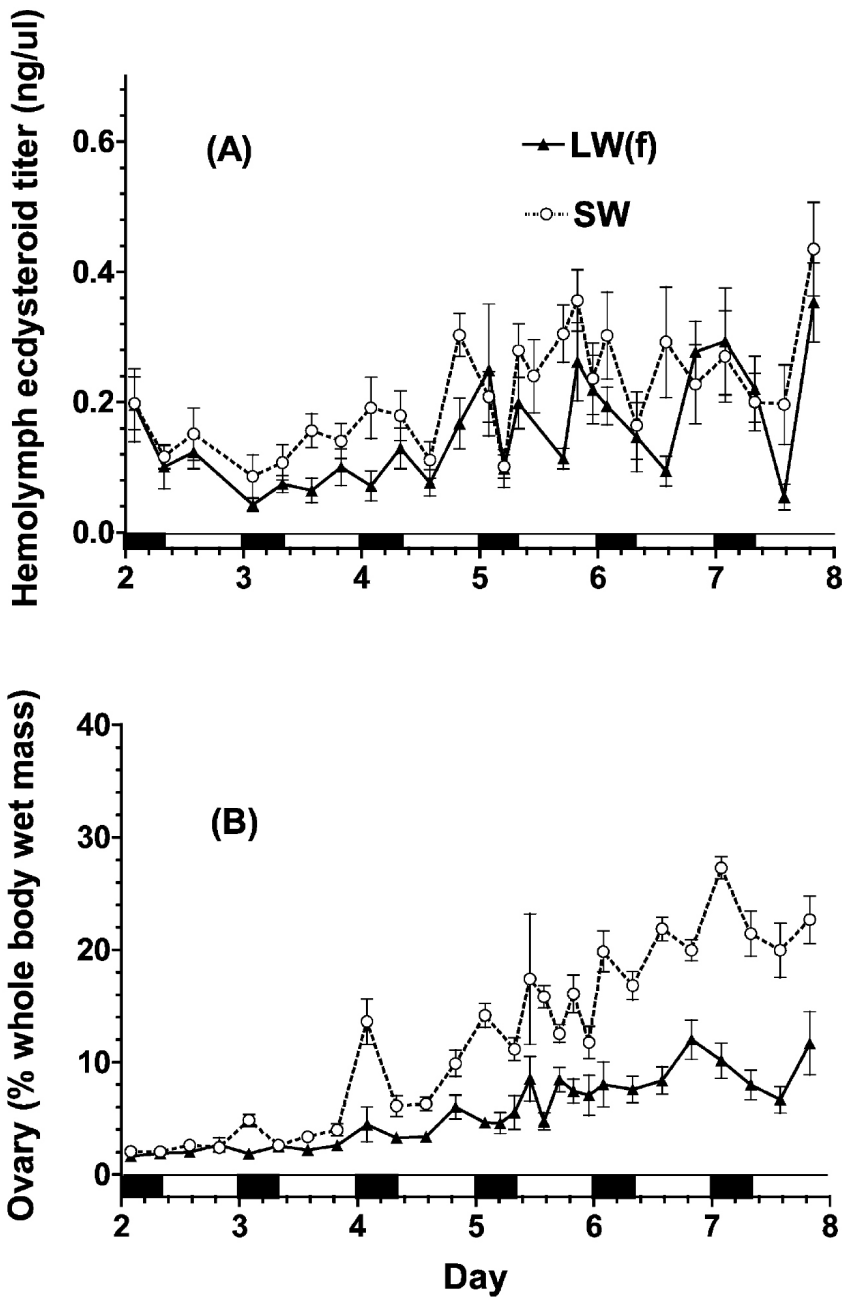

Figure 5. Mean \pm SEM ecdysteroid titer (A) and ovarian wet mass as percentage of whole-body wet mass (B) in $L W(\mathrm{f})$ and SW female G. firmus reared without oviposition substrate. Data for this figure were obtained from the same individuals whose $\mathrm{JH}$ titers are presented in Figure 1. Symbols are the same as those given in Figure 1.

Table 1. Hemolymph volume and activities of JH-degrading enzymes in LW(f) and SW morphs of Gryllus firmus measured early and late in the $16 \mathrm{~h}$ photophase on day 5 of adulthood

\begin{tabular}{llll}
\hline Trait & Morph & $\begin{array}{l}\text { Measurement } \\
\text { Early (lights on) }\end{array}$ & Late (4 h prior to lights off) \\
\hline Hemolymph volume $(\mu \mathrm{l})(\%$ of whole-body wet mass) & LW(f) & $134 \pm 11(16.3 \pm 0.6 \%)$ & $93 \pm 14(12.7 \pm 1.4 \%)$ \\
& SW & $165 \pm 24(22.7 \pm 3.5 \%)$ & $123 \pm 14(14.2 \pm 1.6 \%)$ \\
& LW(f) & $8.4 \pm 0.3$ & $11.9 \pm 1.0^{\mathrm{a}}$ \\
Hemolymph JHE activity & SW & $7.5 \pm 0.3$ & $7.7 \pm 0.4$ \\
& LW(f) & $4.6 \pm 0.2$ & $4.7 \pm 0.3$ \\
Whole-cricket JH-esterase activity & SW & $11.1 \pm 0.6$ & $13.3 \pm 0.5$ \\
& LW(f) & $1.3 \pm 0.1$ & $2.0 \pm 0.1$ \\
Whole-cricket JH-epoxide hydrolase activity & SW & $1.6 \pm 0.2$ & \\
\hline
\end{tabular}

a Units of measurement: hemolymph JHE activity, nmol JH-acid/min/ml; whole-cricket JHE activity, pmol JH acid/min/mg; whole-cricket JH-epoxide hydrolase, pmol JH diol/min/mg. Sample sizes ranged from 7 to 11 for LW(f) and SW morphs. 


\section{Discussion}

Although numerous studies have quantified hemolymph JH titer profiles as a function of insect age (e.g. Riddiford, 1985, Edwards et al., 1995, and Tawfik et al., 2000, and references therein), the potential existence of daily cycles in the JH titer has largely been ignored. Hemolymph sampling has been restricted to the photophase in nearly all studies, and evidence for daily cycles in the JH titer has been reported in only a few cases (Walker and Denlinger, 1980, Elekonich et al., 2001, and Zera and Cisper, 2001; discussed below). The present study represents the most detailed temporal characterization of the JH titer during early adulthood yet undertaken. Hemolymph titers were measured in over 500 individual adult G. firmus, using a well-validated radioimmunoassay (Hunnicutt et al., 1989 and Cisper et al., 2000). Three important results were obtained, each of which will be discussed in more detail below. First, the hemolymph JH titer exhibits a clear morph-dependent diurnal cycle: a large-amplitude daily cycle exists in the flight-capable morph, but, at most, only a low-amplitude cycle is seen in the flightless morph. Second, the hemolymph titer of another group of hormones (ecdysteroids) did not exhibit a large-amplitude daily cycle. Third, the difference between LW(f) and SW morphs in the rise of the $\mathrm{JH}$ titer during the photophase was not due to morph-specific changes in the hemolymph volume or JH degradation.

\subsection{Large-amplitude, morph-dependent hemolymph JH titer in Gryllus firmus}

Zera and Cisper (2001) previously reported a morph-dependent temporal cycle in the JH titer in Gryllus firmus. However, in that study, JH titers were only measured at two time points during the photophase on 2 non-consecutive days. Thus only limited information was obtained on the periodicity or shape of the temporal cycle. The present study clearly demonstrates a large-amplitude ( $>20$-fold increase in mean titer), daily cycle in the hemolymph $\mathrm{JH}$ titer, that occurs in the flight-capable LW(f) morph, but not in the flightless SW morph (Figure 1 and Figure 2). A daily cycle had already begun in LW(f) females on day 2, the first day of adulthood studied, and was observed on each of days 2-8. However, the large-amplitude cycle did not begin until day 5, and was unaffected by the presence/absence of oviposition material.

The large amplitude $\mathrm{JH}$ titer cycle is not a characteristic of all hemolymph hormones, since we did not observe a corresponding cycle for the hemolymph ecdysteroid titer (Figure 2 and Figure 5). Thus, morphdependent titer variation is not simply the result of large scale, morph-dependent changes in hemolymph vol- ume, which would affect the concentration of all components of the hemolymph. Endocrine processes regulating the $\mathrm{JH}$ cycle in the $\mathrm{LW}(\mathrm{f})$ morph may be specific for that hormone. Studies currently in progress also indicate that the morph-dependent $\mathrm{JH}$ titer cycle is not a laboratory artifact. In the field (Gainesville, Florida), the LW(f)-associated, large-amplitude JH titer cycle was observed in both field collected G. firmus and in laboratory stocks of this species (used in the present study) raised in the field (A.J. Zera and Z. Zhao, unpublished data). Furthermore, the JH titer peak occurred at sunset in field-reared crickets, which corresponds to the $\mathrm{JH}$ titer peak at the photophase-scotophase interface in the laboratory (Figure 1 and Figure 2).

In contrast to the clearly-defined, large amplitude $\mathrm{JH}$ titer cycle, which was restricted to the $\mathrm{LW}(\mathrm{f})$ morph, less clearly defined daily cycles of much lower amplitude (2-3-fold change) appeared to exist for the hemolymph JH titer in SW females and for the ecdysteroid titer in both morphs (Figure 1, Figure 2, and Figure 5). These cycles began on days 4-5 of adulthood, with titer peaks occurring at the end of the photophase-beginning of the scotophase, roughly coincident with the large-amplitude JH titer peak in LW(f) females. Such titer cycles are not unexpected since the hemolymph volume contracts during the latter part of the photophase on day 5 ( Table 1). On the other hand, the lowamplitude $\mathrm{JH}$ titer cycle was not seen on days 7-8 in SW females that were allowed to oviposit their eggs ( Figure 2A). This suggests that, at least on some days, the low-amplitude JH cycle in SW females ( Figure 1) may have been influenced by their inability to oviposit eggs. Thus, although LW(f) and SW females clearly differ with respect to the daily cycle in the hemolymph JH titer, it remains uncertain whether the difference is due to presence vs. complete absence of the cycle, or in the amplitude of the cycle.

Numerous examples of diurnal variation in hormone titers have been reported in vertebrates (e.g. Nelson, 1995 and Norris, 1997). An increasing number of cases also are being reported in insects - e.g. hemolymph JH titer in adult Heliothis virescens and Apis mellifera (Ramaswamy et al., 2000 and Elekonich et al., 2001), hemolymph ecdysteroid and prothoracicotropic hormone in Rhodnius prolixus (Vafopoulou and Steel, 1991 and Vafopoulou and Steel, 1996), and adipokinetic hormone and response to adipokinetic hormone in Acheta domesticus and Pyrrhocoris apterus (Das et al., 1993, Maxova et al., 2001, and Kodrik et al., 2003). However, two aspects of the morph-dependent $\mathrm{JH}$ titer diurnal cycle in G. firmus appear to be unique: the amplitude of the cycle and the association of the cycle with only one of several morphs within a species. The amplitudes of the hormone titer cycles in the examples listed above (few fold) were, in general, similar to the low amplitude cy- 
cles seen in G. firmus (JH titer in SW morph; ecdysteroid titer in both morphs), and were much less than that seen for the JH titer in LW(f) females of G. firmus (>20fold change in mean titer; $>100$-fold change in titers in some individuals). This is the largest daily change in the JH titer of which we are aware. Nor do we know of any other example of a morph-dependent diurnal cycle for any hormone in any other species exhibiting complex (phase, caste, or wing/flight-muscle) polymorphism. The hormone titer cycle in G. firmus, which anticipates the beginning of the scotophase, is likely to be an endogenous circadian rhythm, similar to circadian rhythms recently demonstrated for the titers of several insect hemolymph hormones (Vafopoulou and Steel, 1991 and Vafopoulou and Steel, 1996). However, strong evidence supporting this hypothesis requires demonstration of a free-running rhythm of approximately 24 $h$ in constant darkness, which has yet to be done.

\subsection{Inter-individual variation}

As was the case in our previous study (Zera and Cisper, 2001), the JH titer was much more variable in the dispersing $[\mathrm{LW}(\mathrm{f})]$ morph than in the SW morph during the $\mathrm{JH}$ titer peak late in the photophase on days 5-7 ( Figure 3 and Figure 4). Furthermore, in both the present study, and a previous study of Zera and Cisper (2001), there was evidence for a bimodal distribution in the JH titer in the LW(f) morph; most individuals exhibited a JH titer that was higher than that in most SW females, while a few individuals exhibited a low titer that was near the median titer of the SW morph ( Figure 3 and Figure 4). This extensive titer variation in the $\mathrm{LW}(\mathrm{f})$ morph could be due to a variety of causes. For example, there could be multiple, non-synchronous $\mathrm{JH}$ titer daily cycles in the LW(f) morph. Second, variation in the JH titer may regulate or be associated with physiological differences between LW(f) individuals. For example, around day 5, some LW(f) individuals begin to histolyze their flight muscles and are transformed into the flightless LW(h) morph (Zera et al., 1997; see Materials and methods). $\mathrm{LW}(\mathrm{f})$ individuals with a low JH titer, may be in the process of being transformed into the flightless $L W(\mathrm{~h})$ morph which lacks the substantial rise in the JH titer (Zera and Cisper, 2001). Third, JH regulates flight behavior (propensity to fly) and other types of locomotion in insects (Rankin, 1989, Dingle, 1996, and Lin and Lee, 1998). Individuals with different JH titers may be specified to undertake different types of locomotion, such as short-duration, exploratory "ranging" flights (Dingle, 1996), longer duration "migratory" flights, or non-flight-locomotion (e.g. walking/hopping) - see Zera and Cisper (2001) for extensive discussion of the potential role of $\mathrm{JH}$ in morph-specific dispersal in G. firmus.

\subsection{Proximate mechanisms}

The large-amplitude, morph-dependent JH titer cycle in G. firmus raises intriguing questions with respect to the nature of the proximate regulatory mechanisms that are responsible for its existence. Clearly, morphs must differ dramatically with respect to processes involved in $\mathrm{JH}$ titer regulation (e.g. rates of hormone biosynthesis), and likely differ with respect to the control of these regulatory processes by a circadian clock. Furthermore, regulatory mechanisms that control morph determination and development during the juvenile stage (Zera and Denno, 1997), must somehow set the stage for morph-dependent regulation of the JH titer daily cycle, which occurs much later, during the adult stage. This situation is similar to organizational and activational effects of hormones which control the expression of sex-limited traits in adulthood, while sex determination itself occurs in the embryo (Phoenix et al., 1959 and Young et al., 1964).

At present we only can eliminate changes in hemolymph volume and $\mathrm{JH}$ degradation as factors causing the large-magnitude, morph-specific increase in the $\mathrm{JH}$ titer, since these factors neither changed substantially during the photophase, nor changed in a morph-dependent manner (Table 1). The most likely candidate regulator of the large-scale increase in the JH titer is morph-dependent change in JH biosynthesis. Indeed, preliminary results indicate that the rate of $\mathrm{JH}$ biosynthesis is significantly higher in LW(f) vs. SW adult female G. firmus late in the photophase, while rates of biosynthesis are equivalent in the morphs early in the photophase (Z. Zhao and A. J. Zera, unpublished data). Scott et al. (2001) have shown that the rapid increase in the JH titer in a burying beetle, within a few hours after discovery of a carcass, appears to be regulated by increased JH biosynthesis, as well as decreased JH degradation. Although we have eliminated changes in the hemolymph volume and $\mathrm{JH}$ degradation as causal factors in the substantial increase in the JH titer in the LW(f) morph, these factors, especially $\mathrm{JH}$ degradation, might be important in regulating the morph-dependent decrease in the $\mathrm{JH}$ titer during the end of the photophase-beginning of the scotophase. Finally, hemolymph volume decreased significantly in both morphs during the photophase ( Table 1), and thus may contribute to the low-amplitude changes in the hemolymph JH titer (SW morph) and ecdysteroid titer (both morphs) which peaked late in the photophase ( Figure 2 and Figure 5).

\subsection{Implications for general studies of JH endocrinology and specific studies of complex polymorphism}

Results of the present and other studies (Elekonich et al., 2001, Zera and Cisper, 2001, Ramaswamy et al., 2000, 
and Walker and Denlinger, 1980) indicate that diurnal cycles of the hemolymph JH titer may be more common in insects than previously expected. It is important to identify these diurnal cycles, because the failure to do so can result in a highly misleading picture concerning associations between the $\mathrm{JH}$ titer and developmental or reproductive events. For example, a positive correlation was seen between the hemolymph JH titer and ovarian mass in G. firmus females when blood samples were taken early in the photophase, while a negative correlation or no correlation was observed when samples were taken late in the photophase (Zera and Cisper, 2001; Results). It is imperative that future JH titer profile studies involve temporal sampling to determine the extent of any daily titer cycles.

The existence of a morph-dependent diurnal cycle is especially relevant for studies of the endocrine control of complex (e.g. caste, phase, wing/flight muscle) polymorphism. Depending upon the time-of-day at which hemolymph samples are taken, LW(f) G. firmus can have higher, lower, or equivalent $\mathrm{JH}$ titers relative to flightless SW females (Zera and Cisper, 2001; Figure 1 and Figure 2 ). The failure to find endocrine differences between adult morphs in other studies (e.g. Zera et al., 1993 and Tanaka, 1994), may have been the result of the studies having been undertaken during a restricted period of time when endocrine events are similar in the morphs. Thus, it is especially important that characterizations of hormone titers in morphs of a complex polymorphism be based on parallel titer comparisons measured at a variety of points in the photophase and scotophase. Not only might limited diurnal sampling give a misleading picture with respect to morph-specific differences in endocrine characteristics, such a sampling protocol would completely miss a key endocrine difference between morphs, namely a morphdependent daily cycle in the JH titer.

Because the high-amplitude $\mathrm{JH}$ titer diurnal cycle thus far has only been observed in one insect species, it is impossible to know its phylogenetic distribution. If the cycle in the LW(f) morph primarily functions to regulate flight - discussed briefly above, and in more detail in Zera and Cisper (2001) - then morph-dependent, JH titer diurnal cycles may be primarily restricted to dispersal-polymorphic insects, such as crickets, in which flight is restricted to a portion of the day or night. On the other hand, because JH influences so many biological characteristics, morph-dependent daily cycles in the hemolymph JH titer might be common in other cases of complex polymorphism in which morphs differ in characters (e.g. behaviors) that are restricted to specific periods of the day or night.

\section{Acknowledgments}

This study was supported by NSF grant IBN-0130665 to A. J. Zera. Clarity of the manuscript was significantly improved by the thoughtful comments of the two reviewers for Journal of Insect Physiology.

\section{References}

Cisper et al., 2000. G. Cisper, A. J. Zera and D. W. Borst, Juvenile hormone titer and morph-specific reproduction in the wingpolymorphic cricket, Gryllus firmus. Journal of Insect Physiology 46 (2000), pp. 585-596.

Das et al., 1993. S. Das, O. W. Meier and J. Woodring, Diel rhythms of adipokinetic hormone, fat body response, and haemolymph lipid and sugar levels in the house cricket. Physiological Entomology 18 (1993), pp. 233-239.

Dingle, 1996. H. Dingle, Migration: The Biology of Life on the Move., Oxford University Press, Oxford (1996).

Edwards et al., 1995. J. P. Edwards, T. S. Corbitt, H. F. McArdle, J. E. Short and R. J. Weaver, Endogenous levels of insect juvenile hormones in larval, pupal and adult stages of the tomato moth, Lacanobia oleracea. Journal of Insect Physiology 41 (1995), pp. 641-651.

Elekonich et al., 2001. M. M. Elekonich, D. L. Schultz, G. Bloch and G. E. Robinson, Juvenile hormone levels in honey bee (Apis mellifera L.) foragers: foraging experience and diurnal variation. Journal of Insect Physiology 47 (2001), pp. 1119-1125.

Gould, 1977. S. J. Gould, Ontogeny and Phylogeny. Belknap Harvard Press, Cambridge (1977).

Hardie and Lees, 1985. J. Hardie and A. D. Lees, Endocrine control of polymorphism and polyphenism. In: G. A. Kerkut and L. I. Gilbert, Editors, Comprehensive Insect Physiology, Biochemistry and Pharmacology, vol. 8, Pergamon, Oxford (1985), pp. 441-490.

Horn et al., 1976. D. H. S. Horn, J. S. Wilkie, A. Sage and D. O. O'Connor, A high affinity antiserum specific for the ecdysone nucleus. Journal of Insect Physiology 22 (1976), pp. 901-905.

Huang et al., 1994. Z. -Y. Huang, G. E. Robinson and D. W. Borst, Physiological correlates of division of labor independent of age in honey bee colonies. Journal of Comparative Physiology A 174 (1994), pp. 731-739.

Hunnicutt et al., 1989. D. Hunnicutt, Y. Toong and D. W. Borst, A chiral specific antiserum for juvenile hormone. American Zoologist 29 (1989), p. 48a.

Kodrik et al., 2003. D. Kodrik, R. Socha and Z. Syrova, Developmental and diel changes of adipokinetic hormone in CNS and hemolymph of the flightless wing-polymorphic bug, Pyrrhocoris apterus (L.). Journal of Insect Physiology 49 (2003), pp. 53-61.

Lin and Lee, 1998. T. -M. Lin and H. -J. Lee, Parallel control mechanisms underlying locomotor activity and sexual receptivity of the female German cockroach, Blattella germanica (L.). Journal of Insect Physiology 44 (1998), pp. 1039-1051.

Loher et al., 1983. W. Loher, L. Ruzo, F. C. Baker, C. A. Miller and D. A. Schooley, Identification of the juvenile hormone from the cricket, Teleogryllus commodus, and juvenile hormone titre changes. Journal of Insect Physiology 29 (1983), pp. 585-589.

Matsuda, 1987. R. Matsuda, Animal Evolution in Changing Environments with Special Reference to Abnormal Metamorphosis. WileyInterscience, New York (1987).

Maxova et al., 2001. A. Maxova, D. Kodrik, R. Zemek and R. Socha, Diel changes in adipokinetic response and walking activity of Pyrrhocoris apterus (Heteroptera) in relation to physiological status and wing dimorphism. European Journal of Entomology 98 (2001), pp. 433-438.

Nelson, 1995. R. J. Nelson, An Introduction to Behavioral Endocrinology. Sinauer, Sunderland (1995).

Nijhout, 1994. H. F. Nijhout, Insect Hormones. Princeton University Press, Princeton (1994).

Nijhout, 1999. H. F. Nijhout, Control mechanisms of polyphenic development in insects. Bioscience 49 (1999), pp. 181-192. 
Norris, 1997. D. O. Norris, Vertebrate Endocrinology. Academic Press, San Diego (1997).

Phoenix et al., 1959. C. H. Phoenix, R. W. Goy, A. A. Gerall and W. C. Young, Organizing action of prenatally administered testosterone propionate on the tissues mediating female guinea pig. Endocrinology 65 (1959), pp. 369-382.

Ramaswamy et al., 2000. S. B. Ramaswamy, S. Shengqiang, G. N. Mbata, A. Rachinsky, Y. I. Park, L. Crigler, S. Donald and A. Srinivasan, Role of juvenile hormone-esterase in mating-stimulated egg development in the moth Heliothis virescens. Insect Biochemistry and Molecular Biology 30 (2000), pp. 785-791.

Rankin, 1989. M. A. Rankin, Hormonal control of flight. In G. J. Goldsworthy and L. I. Gilbert, Editors, Insect Flight, CRC Press, Boca Raton (1989), pp. 139-163.

Riddiford, 1985. L. M. Riddiford, Hormone action at the cellular level. In G. A. Kerkut and L. I. Gilbert, Editors, Comprehensive Insect Physiology, Biochemistry and Pharmacology, vol. 8, Pergamon, Oxford (1985), pp. 37-84.

Scott et al., 2001. M. P. Scott, S. T. Trumbo, P. A. Neese, W. D. Bailey and R. M. Roe, Changes in biosynthesis and degradation of juvenile hormone during breeding by burying beetles: a reproductive or social role?. Journal of Insect Physiology 47 (2001), pp. 295-302.

Southwood, 1961. T. R. E. Southwood, A hormonal theory of the mechanism of wing polymorphism in Heteroptera. Proceedings of Royal Entomological Society, London 36 (1961), pp. 63-66.

Tanaka, 1994. S. Tanaka, Endocrine control of ovarian development and flight muscle histolysis in a wing dimorphic cricket, Modicogryllus confirmatus. Journal of Insect Physiology 40 (1994), pp. 483-490.

Tawfik et al., 2000. A. I. Tawfik, K. Treiblmayr, A. Hassanali and E. O. Osir, Time-course haemolymph juvenile hormone titres in solitarious and gregarious adults of Schistocerca gregaria and their relation to pheromone emission, CA volumetric changes and oocyte growth. Journal of Insect Physiology 46 (2000), pp. 1143-1150.

Trumbo et al., 1995. S. T. Trumbo, D. W. Borst and G. E. Robinson, Rapid elevation of juvenile hormone titer during behavioral assessment of the breeding resource by the burying beetle, Nicrophorus orbicollis. Journal of Insect Physiology 41 (1995), pp. 535-543.

Vafopoulou and Steel, 1991. X. Vafopoulou and C. G. Steel, Circadian regulation of synthesis of ecdysteroids by prothoracic glands of the insect Rhodnius prolixus: Evidence for a dual oscillator system. General and Comparative Endocrinology 83 (1991), pp. 27-34.

Vafopoulou and Steel, 1996. X. Vafopoulou and C. G. Steel, The insect neuropeptide prothoracicotropic hormone is released with a daily rhythm: Re-evaluation of its role in development. Proceedings of the National Academy of Sciences USA 93 (1996), pp. 3368-3372.

Veazy et al., 1976. J. N. Veazy, C. A. R. Kay, T. J. Walker and W. $\mathrm{H}$. Whitcomb, Seasonal abundance, sex ratio, and macroptery of field crickets in northern Florida. Annals of the Entomological Society of America 69 (1976), pp. 374-380.

Walker and Denlinger, 1980. G. P. Walker and D. L. Denlinger, Juvenile hormone and moulting hormone titres in diapauseand non-diapause destined flesh flies. Journal of Insect Physiology 26 (1980), pp. 661-664.
Wigglesworth, 1961. V. B. Wigglesworth, Insect polymorphisma tentative synthesis. In J. S. Kennedy, Editor, Insect Polymorphism, Royal Entomological Society, London (1961), pp. 103-113.

Wyatt and Davey, 1996. G. R. Wyatt and K. G. Davey, Cellular and molecular actions of juvenile hormone. II. Roles of juvenile hormone in adult insects. Advances in Insect Physiology 26 (1996), pp. 1-155.

Yadlowski, 1994. D. E. Yadlowski, Juvenile hormone esterase and correlated responses to selection: The physiological basis of wing dimorphism in the sand cricket, Gryllus firmus (Orthoptera: Gryllidae)., Department of Biology, Concordia University, Montreal, Canada (1994).

Young et al., 1964. W. C. Young, R. W. Goy and C. H. Phoenix, Hormones and sexual behavior. Science 143 (1964), pp. 212-218.

Zera and Bottsford, 2001. A. J. Zera and J. Bottsford, The endocrine-genetic basis of life-history variation: Relationship between the ecdysteroid titer and morph-specific reproduction in the wing-polymorphic cricket, Gryllus firmus. Evolution 55 (2001), pp. 538-549.

Zera and Cisper, 2001. A. J. Zera and G. Cisper, Genetic and diurnal variation in the juvenile hormone titer in a wing-polymorphic cricket: Implications for the evolution of life histories and dispersal. Physiological and Biochemical Zoology 74 (2001), pp. 293-306.

Zera and Denno, 1997. A. J. Zera and R. F. Denno, Physiology and ecology of dispersal polymorphism in insects. Annual Review of Entomology 42 (1997), pp. 207-231.

Zera and Huang, 1999. A. J. Zera and Y. Huang, Evolutionary endocrinology of juvenile hormone esterase: Functional relationship with wing polymorphism in the cricket, Gryllus firmus. Evolution 53 (1999), pp. 837-847.

Zera and Harshman, 2001. A. J. Zera and L. Harshman, Physiology of life history trade-offs in animals. Annual Review of Ecology and Systematics 32 (2001), pp. 95-126.

Zera and Holtmeier, 1992. A. J. Zera and C. L. Holtmeier, In vivo and in vitro degradation of juvenile hormone-III in presumptive long-winged and short-winged Gryllus rubens. Journal of Insect Physiology 38 (1992), pp. 61-74.

Zera and Larsen, 2001. A. J. Zera and A. Larsen, The metabolic basis of life history variation: Genetic and phenotypic differences in lipid reserves among life history morphs of the wing-polymorphic cricket, Gryllus firmus. Journal of Insect Physiology 47 (2001), pp. 1147-1160.

Zera et al., 1993. A. J. Zera, C. A. Borcher and S. B. Gaines, Juvenile hormone degradation in adult wing morphs of the cricket, Gryllus rubens. Journal of Insect Physiology 39 (1993), pp. 845-856.

Zera et al., 1997. A. J. Zera, J. Sall and K. Grudzinski, Flight-muscle polymorphism in the cricket Gryllus firmus: Muscle characteristics and their influence on the evolution of flightlessness. Physiological Zoology 70 (1997), pp. 519-529.

Zera et al., 1989. A. J. Zera, C. Strambi, K. C. Tiebel, A. Strambi and M. A. Rankin, Juvenile hormone and ecdysteroid titers during critical periods of wing morph determination in Gryllus rubens. Journal of Insect Physiology 35 (1989), pp. 501-511. 